\title{
Pratiques
}

Linguistique, littérature, didactique

187-188 | 2020

Enseignement du texte littéraire dans l'espace

francophone: pratiques, formation, recherche

\section{Le noyau dur des pratiques d'enseignement de la poésie au secondaire québécois}

The core of poetry teaching practices in Québec highschools

\section{Judith Émery-Bruneau}

\section{(2) OpenEdition \\ Journals}

Édition électronique

URL : https://journals.openedition.org/pratiques/8907

DOI : $10.4000 /$ pratiques.8907

ISSN : 2425-2042

Éditeur

Centre de recherche sur les médiations (CREM)

Référence électronique

Judith Émery-Bruneau, «Le noyau dur des pratiques d'enseignement de la poésie au secondaire québécois », Pratiques [En ligne], 187-188 | 2020, mis en ligne le 12 décembre 2020, consulté le 21 juillet 2021. URL : http://journals.openedition.org/pratiques/8907 ; DOI : https://doi.org/10.4000/ pratiques. 8907

Ce document a été généré automatiquement le 21 juillet 2021.

(c) Tous droits réservés 


\title{
Le noyau dur des pratiques d'enseignement de la poésie au secondaire québécois
}

\author{
The core of poetry teaching practices in Québec highschools
}

\author{
Judith Émery-Bruneau
}

1 Lorsque le pianiste et compositeur de jazz Bill Evans a décrit son intérêt pour le bouddhisme, bien qu'il s'agissait d'une pratique culturelle qui ne faisait pas partie de son quotidien, il a répondu ceci (nous ajoutons l'italique) :

I don't pretend to understand it. I just find it comforting - and very similar to jazz. Like jazz, you can't explain it to anyone without losing the experience. That's why it bugs me when people try to analyze jazz as an intellectual theorem. It's not. It's feeling. ${ }^{1}$

Qu'elles soient philosophiques, d'un genre musical ou littéraire - comme la poésie -, être exposés à de telles pratiques culturelles, s'autoriser à vivre des expériences variées sans nous sentir obligés de devoir toujours adopter un regard analytique ou encore les comparer à d'autres expériences culturelles ou littéraires qui nous ont marqués, cela permet d'apprendre sur soi et sur le monde, et, comme sujet, de transformer peu à peu notre rapport à cette pratique. Cependant, la poésie n'est pas qu'émotion - comme le jazz d'ailleurs («it's not just feeling», pour réagir aux propos de B. Evans). Pour des lecteurs réels, particulièrement en contexte scolaire, des savoirs et des outils peuvent s'avérer nécessaires pour comprendre et apprécier un poème, certes, mais également pour arriver à mieux justifier leurs interprétations ou nommer ce qu'il leur fait vivre. Ces savoirs contribuent à produire un discours sur ce qui fait évènement lorsque, comme lecteurs, nous nous retrouvons devant un poème ou assistons à une performance poétique. S'engager dans une telle réflexion sur l'expérience de la lecture littéraire ouvre la voie aux manifestations de la réflexivité sur cette pratique sociale ou sur l'objet auquel nous sommes exposés.

3 La présente recherche trouve ses origines dans ces réflexions : nous pensons qu'il est essentiel, utile et urgent de renouer avec la poésie en contexte scolaire étant donné 
qu'il s'agit d'un des genres littéraires qui déstabilise le plus les enseignants de français du secondaire (Bergeron, 2016; Duval, 2004 ; Massart-Laluc, 2010). Nous avons donc voulu connaitre les pratiques ordinaires d'enseignement de la poésie au secondaire québécois, lesquelles n'avaient pas encore été documentées à ce jour ${ }^{2}$, afin de mieux comprendre comment la poésie est lue et étudiée en classe. Une vingtaine d'enseignants du secondaire québécois, répartis sur les deux cycles d'enseignement, ont participé à notre recherche. Outre le fait d'avoir mené des entretiens pour connaitre leurs conceptions ${ }^{3}$ de la poésie et de son enseignement, nous avons observé et filmé leur séquence d'enseignement de la poésie. Nous avons ensuite produit des synopsis (Schneuwly \& Dolz, 2009) de ces séquences (composée de 3 à 13 séances, selon les cas) dans le but d'analyser les objets enseignés, les corpus de textes poétiques, les tâches données aux élèves, les genres d'activités scolaires (Aeby-Daghé, 2014) privilégiées par les enseignants, ainsi que les gestes de régulation et d'institutionnalisation observés pendant l'action didactique.

Pour cette contribution, nous avons choisi de nous concentrer sur les trames prototypiques qui se dégagent des synopsis, c'est-à-dire la macrostructure des séquences d'enseignement qui organise les contenus, ainsi que sur le noyau dur des savoirs enseignés et mobilisés dans ces séquences sur la poésie. Particulièrement révélateurs pour cerner ce qui compose le cœur des pratiques d'enseignement de la poésie, ces éléments ont permis d'identifier les points de complémentarité et de rupture entre l'étude de savoirs déclaratifs sur la poésie et l'analyse de poèmes. Nos résultats montrent que la poésie est peu ou prou travaillée en classe pour vivre et faire vivre des expériences poétiques (Brillant-Rannou, 2010); les pratiques visent plutôt à étudier des notions littéraires dans le but de les repérer dans les poèmes ou de les reproduire à l'écrit.

\section{Cristallisation, sédimentation ou éclatement des pratiques d'enseignement de la poésie?}

5 La poésie est prescrite et enseignée à tous les degrés de la scolarité obligatoire québécoise, du primaire (6-12 ans), au $1^{\mathrm{er}}$ cycle du secondaire (12-14 ans), puis au $2^{\mathrm{e}}$ cycle (14-17 ans). La progression prescrite par le ministère de l'Éducation repose sur les genres de textes poétiques à faire lire, écrire ou écouter. Par exemple, les élèves doivent lire des poèmes ayant une trame narrative ou descriptive en $1^{\text {re }}$ année du secondaire et faire des jeux poétiques (comme le cadavre exquis) ; lire des fables en $2^{\mathrm{e}}$; en $3^{\mathrm{e}}$, ils doivent lire des poèmes lyriques et écrire une chanson ou transformer un poème original ; les deux dernières années $\left(4^{e}\right.$ et $\left.5^{\mathrm{e}}\right)$, il est prescrit de leur faire lire des poèmes lyriques et engagés, classiques et contemporains, d'écrire un poème engagé et d'écouter des slam, ainsi que des poèmes ou chansons engagés (Ministère de l'Éducation, du Loisir et du Sport du Québec, 2011, p. 37-42). Il est en outre souligné dans les programmes qu'au $1^{\text {er }}$ cycle du secondaire, les élèves doivent se familiariser avec la poésie et apprendre à jouer avec la langue : c'est un rapport ludique et participatif à la poésie qui est encouragé. Au $2^{e}$ cycle, on prescrit d'inviter les élèves à réfléchir sur le monde et la culture : un rapport plus distancié à l'analyse de poèmes devrait alors s'installer. Or, quels arrimages observe-t-on entre les compétences lectorales travaillées en classe et les notions de poésie enseignées ? Ces savoirs, si l'on se fie aux pratiques déclarées des enseignants que nous avons interrogés dans le cadre 
d'entretiens semi-dirigés, sont le plus souvent les mêmes d'une année à l'autre, peu importe le genre poétique travaillé (règles de versification, figures de style, poèmes à forme fixe, écriture d'imitation...; Émery-Bruneau, 2018a). Et cette réalité n'est pas propre au Québec ; c'est aussi le cas en France, comme l'ont souligné N. Brillant-Rannou et C. Boutevin $(2018$, p. 14) : "Les approches sont bien souvent figées: écriture d'imitation ou à contraintes, peu de lecture extensive, obsession formelle de la versification et surtout très peu de lecture subjective ».

Plusieurs praticiens et chercheurs l'ont observé : élèves et enseignants ont un rapport inconfortable à la poésie et se sentent plus ou moins à l'aise de la travailler en classe (Bergeron, 2016; Duval, 2004 ; Massart-Laluc, 2010). Serait-ce ce malaise vis-à-vis de l'enseignement de la poésie qui poussa J.-L. Dufays, M. Lisse et C. Meurée $(2009$, p. 76$)$ à critiquer le travail des enseignants en soulignant que «trop souvent, la poésie est abordée tantôt de manière techniciste, tantôt à l'inverse sans aucune méthode » ? D'ailleurs, pour reprendre les mots de V. Martinez (2018, p. 238), faudrait-il « revoir la chaîne du savoir qui forme les enseignants et les lecteurs à la poésie, en "défragmentant" et "détechnicisant" le rapport à ce genre "? Or, alors que la lecture expliquée semble être une méthode d'enseignement de la poésie qui s'est cristallisée, les pratiques traditionnelles de récitation et d'écriture d'imitation de morceaux choisis, qui ont marqué l'histoire de l'enseignement de la poésie (Chervel, 2006), se sont quant à elles sédimentées, car les pratiques actuelles d'enseignement de la poésie n'ont, certes, pas effacé les anciennes, mais s'y sont superposées, c'est-à-dire que les pratiques traditionnelles se sont transformées. Par exemple, au Québec, la récitation de morceaux choisis s'est amalgamée à la récitation de slam (Émery-Bruneau, 2018b): les mêmes critères d'évaluation sont utilisés (articulation, débit, volume...), pour des genres pourtant très différents, à commencer par l'intention de communication des locuteurs. Mais si « c'est sans doute à l'école qu'on lit le plus de poésie et, une fois hors d'elle, on n'en lit guère » (Canvat \& Legros, 1997, p. 5), car la poésie ne fait pas partie des lectures privées des jeunes (Baudelot, Cartier \& Detrez, 1999), et s'il n'y a, en règle générale, qu'au plus une seule séquence d'enseignement de la poésie qui est mise en place par année au secondaire québécois, alors que fait-on avec la poésie à l'école ? Qu'enseignet-on d'une année à l'autre ? Comment l'enseigne-t-on? Et dans quel but?

\section{La poésie, un objet d'enseignement ?}

Deux didacticiens de la littérature de la première génération défendaient qu'un "véritable enseignement de la poésie devrait viser à former des lecteurs connaisseurs et pas seulement des amateurs, et que de tels lecteurs ont besoin de connaissances même sur des œuvres qu'ils n'ont pas lues " (Canvat \& Legros, 1997, p. 26). Selon eux, les pratiques d'enseignement de la poésie devraient ainsi miser sur la transmission trois savoirs :

- sociaux (le champ littéraire et ses conflits de valeurs, le paratexte, l'image des poètes, les modes de lecture, etc.) ;

- techniques (savoirs syntaxiques, sémantiques, métriques, génériques, rhétoriques, narratologiques - le cas échéant -, typographiques, etc.);

- et historiques (contexte de production, courant littéraire, etc.).

Or, pour rappeler la réflexion soulevée par S. Martin (2018a), comment ne plus se laisser prendre par les injonctions traditionnelles de la définition de la poésie et 
déplacer notre focale vers celles plus récentes de la réception? Il s'agirait alors de déplacer notre intérêt du lecteur implicite ou virtuel au lecteur réel et, conséquemment, du «texte de l'œuvre » au «texte du lecteur » (Mazauric, Fourtanier \& Langlade, 2011, p. 19-20). Dans cette perspective, s'intéresser à la réception permettrait de concevoir autrement la poésie et sa didactique. Cela dit, au moment où K. Canvat et $\mathrm{G}$. Legros écrivaient leur article, le champ de la didactique de la littérature était émergeant ${ }^{4}$ et, forcément, il y a 25 ans, encore peu de recherches avaient porté spécifiquement sur l'enseignement et l'apprentissage de la poésie au secondaire. Mais nous y sommes-nous davantage intéressés depuis? M. Favriaud a encore dénoncé récemment le fait que «la didactique de la poésie a connu trente ans de basses eaux: ainsi la revue Pratiques n'a-t-elle pas abordé ce champ depuis $1997 »$ (2018, p. 77). Qui plus est, le concept du "sujet lecteur $"^{5}$, apparu il y a une quinzaine d'années dans un colloque tourné vers l'expérience sensible et réflexive de lecteurs réels, s'est depuis taillé une place notoire dans notre champ comme l'a rappelé B. Louichon (2011), bien qu'il demeure plutôt récent dans l'histoire de notre discipline. On ne peut alors s'étonner que ces quelques réflexions didactiques n'aient pas encore trouvé d'ancrage concret dans les pratiques d'enseignement.

9 Aujourd'hui, pour les didacticiens qui se sont intéressés à cet objet, l'enseignement de la poésie renverrait non seulement à l'étude de savoirs scolaires, mais aussi aux expériences poétiques vécues et partagées, à la fois par les enseignants et leurs élèves, pour permettre à ces derniers de se constituer en tant que «sujet ». En plus des savoirs sociaux, techniques et historiques à mobiliser, comme le proposaient K. Canvat et G. Legros (1997), cette «lecture scolaire doit aussi être conçue comme une ouverture vers une lecture personnelle qui saurait prendre "patience et longueur des temps" " (Massol, 2016, p. 133). Ainsi s'ajoutent les pratiques langagières réelles, concrètes, directes des sujets lecteurs, scripteurs et performeurs qui mobilisent diverses ressources (épistémiques, cognitives, psychoaffectives, socioculturelles, axiologiques, pragmatiques) pour habiter les poèmes et rendre compte de leur rapport à la poésie. Car, comme l'ont rappelé N. Brillant-Rannou et C. Petit $(2015$, p. 161) : « Du point de vue de la réception, le poème tel qu'on le considère aujourd'hui relève d'un vécu global pour la personne qui le reçoit: il est à lire, dire, entendre et accueillir en tant qu'expérience empirique, sensorielle et émotionnelle, comme une chance d'évènement ». L'expérience poétique serait, comme l'a décrit V. Martinez (2018), un évènement de langage, de sens, de surprise, d'étonnement, d'existence et de transformation de soi. Mais est-ce que l'enseignement de savoirs scolaires, techniques, empêcherait les élèves de vivre une telle expérience de "sujet»? Pourrions-nous penser à un enseignement de la poésie qui s'inscrirait en équilibre entre ces savoirs scolaires transmis par les enseignants, les expériences poétiques vécues par les sujets et le recours à ces savoirs pour analyser et expliquer leurs expériences?

Notre réflexion s'articule ici autour des questions suivantes : comment organise-t-on les savoirs et les pratiques poétiques dans les séquences d'enseignement de la poésie au secondaire québécois aujourd'hui? Quels savoirs ou outils de lecture de poèmes mobilise-t-on dans ces séquences d'enseignement? Quels lecteurs de poésie forme-t-on réellement? Ainsi cette contribution vise-t-elle à dégager les régularités notionnelles (savoirs) et séquentielles (démarches), car avant de prescrire ou même d'évaluer des pratiques, il nous apparait fondamental de connaitre à priori les pratiques ordinaires d'enseignement de la poésie et de comprendre pourquoi il en est ainsi. 


\section{Méthodologie pour analyser les pratiques d'enseignement de la poésie}

11 Nous postulons, à l'instar de B. Schneuwly (dans la préface de l'ouvrage dirigé par Thévenaz-Christen et al., 2014, p. 13), que faire de la didactique, c'est, entre autres, "décrire et expliquer ce qui s'enseigne dans des classes ordinaires, au jour le jour, indépendamment de toute intervention didactique ». C'est dans cette posture épistémologique que s'inscrit la présente recherche.

\section{Données collectées : séquences d'enseignement de la poésie}

La collecte de données s'est organisée autour des pratiques ordinaires d'enseignement au secondaire québécois. Notre unité d'analyse repose sur la séquence d'enseignement annuelle de poésie créée et mise en place par chaque enseignant qui avait reçu pour seule consigne d'enseigner la poésie à leur manière: nous en avons observé le déploiement in situ, tout en la filmant pour ensuite la transcrire (verbatim) afin d'en faciliter le traitement. Rappelons que nous avions préalablement mené des entretiens semi-dirigés sur les conceptions et pratiques déclarées des 20 enseignants participant à notre recherche et dont les résultats ont fait l'objet d'autres articles (Émery-Bruneau, 2018a, 2018b). Pour la présente contribution, nous nous concentrons sur 10 séquences d'enseignement (deux par degré scolaire), animées par autant d'enseignants, ce qui représente quatre séquences pour le $1^{\mathrm{er}}$ cycle et six séquences pour le $2^{\mathrm{e}}$ cycle. La durée des séquences varie, certaines ne reposant que sur trois séances de 75 minutes alors que d'autres s'échelonnent jusqu'à 13 séances de 60 minutes. L'ensemble des 10 séquences d'enseignement renvoie à 59 séances filmées (voir tableau 1 pour une description générale de ces séquences).

\section{Analyse et interprétation des données : le synopsis}

13 Le principal outil d'analyse et d'interprétation des données retenues a été le synopsis, outil créé par le Grafe (Schneuwly \& Dolz, 2009), que nous avons adapté en fonction de nos objectifs de recherche. Rappelons que cet outil permet d'organiser et de hiérarchiser chaque séquence pour en obtenir une vue d'ensemble afin de dégager des régularités notionnelles (savoirs et pratiques enseignés) et séquentielles (démarches privilégiées par les enseignants). Cet outil offre une analyse extrêmement détaillée de la construction et de la transformation de l'objet enseigné. Ainsi, les synopsis produits nous ont-ils permis d'observer l'action didactique à travers :

- le découpage et l'élémentarisation de l'objet, soit les savoirs, corpus et pratiques enseignés ;

- le temps de travail sur ces objets ; les tâches et consignes données ;

- les interactions sur l'objet enseigné ; l'utilisation du métalangage pour parler de l'objet ;

- les gestes didactiques (régulation, institutionnalisation, mémoire didactique, etc.) ;

- les formes sociales de travail (individuel, en dyade, etc.) ;

- les genres d'activités scolaires (travail sur la compréhension, débat interprétatif, etc.) ;

- le matériel utilisé. 
Pour mieux dégager les mouvements de l'action didactique, le découpage (organisation et hiérarchisation des objets élémentés) se déplie en quatre niveaux :

- les niveaux 1 et 2 ( $n$ et $n-n)$ constituent la macrostructure qui permet de dégager les trames prototypiques.

- Le niveau 1 renvoie aux pratiques poétiques et langagières travaillées (ex. : Lecture de poèmes d'amour).

- Le niveau 2 cerne la pratique, ainsi que le texte travaillé (ex. : Lecture à voix haute de Pour toi mon amour de Prévert ; Lecture individuelle de Notre sentier de Félix Leclerc). L'analyse de ce niveau nous a permis de dégager les trames prototypiques et de déceler des finalités fondatrices régulant les séquences d'enseignement.

- le niveau 3 (n-n-n) présente la plus grande densité de données, puisqu'on y décrit les consignes, les questions, les (re)formulations, etc. pour cerner ce qui se passe dans la classe en fonction de l'activité de l'enseignant (par exemple: solliciter les réactions suscitées, analyser le poème vers par vers).

- le niveau 4 (n-n-n-n) permet de cerner l'élémentarisation de l'objet enseigné. Il s'agit du découpage le plus microscopique du synopsis qui, parfois, ne renvoie qu'à quelques secondes d'enseignement dans la séquence (par exemple: figure de style, courant littéraire, biographie de l'auteur). L'analyse de ce niveau nous a permis de cerner le noyau dur de l'enseignement de la poésie, en termes de savoirs et de pratiques.

15 À ces niveaux s'ajoute le niveau 0 , lequel renvoie à un moment de rupture de l'activité didactique, occasionné par exemple par un problème de gestion de la classe (discipliner un élève turbulent), un problème logistique (installer du matériel audiovisuel ou gérer les problèmes de connexion à Youtube pour faire écouter une chanson), des consignes pour une sortie scolaire à venir, etc.

Ces niveaux offrent comme avantage de travailler avec différents grains d'analyse, ce qui permet de mieux mettre en perspective les analyses microscopiques d'un moment précis analysé, par exemple, les 12 minutes d'enseignement sur les formes fixes en poésie, inscrite dans une séquence de huit heures sur la chanson engagée. Au moment de produire nos synopsis, quatre principes ont toujours été respectés, à savoir: le centrage sur l'activité de l'enseignant et son point de vue (comment); les dimensions de l'objet travaillé (quoi); l'objectif de l'enseignant (pourquoi); les moyens utilisés pour y parvenir (comment).

\section{Pratiques d'enseignement de la poésie au secondaire : nos résultats}

17 Précisons que les résultats présentés ici reposent sur une dizaine de séquences d'enseignement observées, certes représentatives des 20 cas $^{6}$ préalablement interrogés sur leurs pratiques déclarées et leurs conceptions de la poésie. Les séquences observées représentent les pratiques ordinaires de ces enseignants dans la mesure où la seule demande qu'ils ont reçue était d'enseigner leur séquence (contenu et méthode) comme ils ont l'habitude de le faire chaque année. Les données ont subi un traitement qualitatif, ce qui a permis de dégager des tendances (et non des généralisations à l'ensemble des enseignants québécois). Malgré ce nombre plutôt restreint, nous avons atteint une saturation des données suffisamment satisfaisante ${ }^{7}$ pour dégager un noyau dur de savoirs et pratiques enseignés et une trame prototypique, soit des suites 
d'activités qui présentent ces savoirs et pratiques dans le même ordre, de façon relativement stable et répétitive. Soulignons que, pour ce faire, nous avons ici fait abstraction du facteur " progression », qui n'a pas fait l'objet d'une analyse fine pour la présente contribution, mais que nous avons traité ailleurs (Émery-Bruneau, 2018a).

\section{Description générale des séquences}

Le tableau suivant présente une synthèse des séquences en termes de durée ${ }^{8}$, ainsi que les genres travaillés qui les ont structurées. Le temps filmé renvoie aux données recueillies lors des captations filmées 9 . Soulignons qu'un prénom fictif commençant par la lettre du degré scolaire dans lequel elles enseignent a été donné aux enseignantes (exemple : $\underline{\text { Diane }}=$ deuxième année du secondaire), afin de faciliter l'organisation des données en fonction des degrés.

Tableau 1 : description globale des séquences analysées

\begin{tabular}{|c|c|c|c|c|c|c|c|c|c|c|}
\cline { 2 - 11 } \multicolumn{1}{c|}{} & Pathy & Pauline & Diane & Denise & Tessa & Tina & Quiana & Queenie & Cindy & Cynthia \\
\hline $\begin{array}{c}\text { Nb } \\
\text { séances }\end{array}$ & 6 & 4 & 9 & 3 & 4 & 13 & 5 & 8 & 4 & 3 \\
\hline $\begin{array}{c}\text { Durées } \\
\text { séances }\end{array}$ & $75 \mathrm{~min}$ & $75 \mathrm{~min}$ & $75 \mathrm{~min}$ & $75 \mathrm{~min}$ & $60 \mathrm{~min}$ & $60 \mathrm{~min}$ & $45 \mathrm{~min}$ & $45 \mathrm{~min}$ & $75 \mathrm{~min}$ & $50 \mathrm{~min}$ \\
\hline $\begin{array}{c}\text { Temps } \\
\text { filmé }\end{array}$ & $7 \mathrm{~h} 30$ & $5 \mathrm{~h} 05$ & $11 \mathrm{~h} 28$ & $3 \mathrm{~h} 49$ & $2 \mathrm{~h} 57$ & $12 \mathrm{~h} 09$ & $2 \mathrm{~h} 52$ & $5 \mathrm{~h} 13$ & $5 \mathrm{~h} 00$ & $2 \mathrm{~h} 16$ \\
\hline Genres & fable & poésie & poésie & slam & $\begin{array}{c}\text { poème } \\
\text { lyrique }\end{array}$ & chanson & haiku & slam & chanson & $\begin{array}{c}\text { poésie } \\
\text { engagée }\end{array}$ \\
\hline $\begin{array}{c}\text { Nb } \\
\text { textes }\end{array}$ & 9 & 9 & 16 & 6 & 1 & 29 & 37 & 19 & 3 & 1 \\
\hline
\end{tabular}

Une conception plutôt large des genres associés à la poésie s'observe, par exemple, en incluant le slam et la chanson contemporaine tout autant que des genres plus traditionnels tels que la fable, le poème lyrique, le haïku ou le poème engagé. Certaines séquences ne portent pas sur un genre spécifique, comme celles de Pauline et de Diane, qui ont organisé l'ensemble de leur séquence autour de la lecture et de l'écriture de poèmes de genres variés. Par ailleurs, la durée des séquences observées est très variable, de même que le nombre de textes travaillés. Les deux plus longues séquences (Diane et Tina) sont néanmoins celles animées par les deux enseignantes qui semblent les plus à l'aise avec la poésie, la première parce qu'elle est écrivaine, slameuse et poète; la seconde, parce qu'elle a une formation initiale de trois ans en études littéraires, suivie d'une maitrise en éducation alors que les enseignants au secondaire québécois ont, en règle générale, l'équivalent d'un an de formation universitaire en littérature, un an en linguistique et deux années en sciences de l'éducation. Ces deux enseignantes sont aussi celles qui animent des séquences marquées de trames prototypiques qui se distinguent en partie de celles de leurs pairs, comme nous le décrivons plus loin.

Le tableau 2 qui suit présente une synthèse des macrostructures des séquences, réparties en fonction de trois ensembles : les moments de transmission de savoirs sur la poésie, le temps de travail lié à des pratiques poétiques ou langagières (lecture, écriture, écoute), les moments de rupture de l'action didactique (niveau 0). 
Tableau 2 : macrostructure des séquences

\begin{tabular}{|c|c|c|c|c|c|c|c|c|c|c|}
\hline & Pathy & Pauline & Diane & Denise & Tessa & Tina & Quiana & Queenie & Cindy & Cynthia \\
\hline \multirow{2}{*}{ Savoirs } & $1 \mathrm{~h} 15 \mathrm{~m} 15$ & $18 \mathrm{~m} 25$ & $1 \mathrm{~h} 28 \mathrm{~m} 35$ & $24 \mathrm{~m} 35$ & & $2 \mathrm{~h} 03 \mathrm{~m} 55$ & $21 \mathrm{~m} 55$ & $1 \mathrm{~h} 06 \mathrm{~m} 40$ & $49 \mathrm{~m} 40$ & $29 \mathrm{~m} 10$ \\
\hline & $16,7 \%$ & $6,0 \%$ & $12,9 \%$ & $10,7 \%$ & & $17,2 \%$ & $12,7 \%$ & $21,3 \%$ & $13,3 \%$ & $21,4 \%$ \\
\hline \multirow{2}{*}{ Lecture } & Ih $58 \mathrm{~m} 50$ & $36 \mathrm{~m} 55$ & $3 \mathrm{~h} 52 \mathrm{~m} 45$ & & & $5 \mathrm{~h} 34 \mathrm{~m} 30$ & $1 \mathrm{~h} 03 \mathrm{~m} 20$ & & $1 \mathrm{~h} 32 \mathrm{~m} 15$ & $39 \mathrm{~m} 25$ \\
\hline & $26,4 \%$ & $12,1 \%$ & $33,8 \%$ & & & $46,2 \%$ & $36,7 \%$ & & $24,7 \%$ & $28,9 \%$ \\
\hline \multirow[b]{2}{*}{ Écriture } & $1 \mathrm{~h} 55 \mathrm{~m} 55$ & $40 \mathrm{~m} 40$ & $17 \mathrm{~m} 35$ & $6 \mathrm{~m} 15$ & $2 \mathrm{~h} 25 \mathrm{~m} 40$ & & $38 \mathrm{~m} 00$ & & & \\
\hline & $25,7 \%$ & $13,3 \%$ & $2,5 \%$ & $2,7 \%$ & $82,5 \%$ & & $22,1 \%$ & & & \\
\hline \multirow[b]{2}{*}{ Écoute } & & $7 \mathrm{~m} 35$ & $12 \mathrm{~m} 00$ & $28 \mathrm{~m} 25$ & $4 \mathrm{~m} 05$ & $26 \mathrm{~m} 05$ & & $2 \mathrm{~h} 30 \mathrm{~m} 50$ & & \\
\hline & & $2,5 \%$ & $1,7 \%$ & $12,4 \%$ & $2,3 \%$ & $3,6 \%$ & & $48,1 \%$ & & \\
\hline \multirow{2}{*}{ Niveau 0} & $2 \mathrm{~h} 23 \mathrm{~m} 15$ & $3 \mathrm{~h} 22 \mathrm{~m} 05$ & $5 \mathrm{~h} 38 \mathrm{~m} 15$ & $2 \mathrm{~h} 50$ & $28 \mathrm{~m} 20$ & $3 \mathrm{~h} 58 \mathrm{~m} 20$ & $50 \mathrm{~m} 10$ & $1 \mathrm{~h} 37 \mathrm{~m} 00$ & $1 \mathrm{~h} 38 \mathrm{~m} 20$ & Ih07m45 \\
\hline & $31,2 \%$ & $66,1 \%$ & $49,1 \%$ & $74,2 \%$ & $15,2 \%$ & $33,0 \%$ & $28,5 \%$ & $30,6 \%$ & $32,7 \%$ & $49,7 \%$ \\
\hline
\end{tabular}

21 Nous observons que le niveau 0 est élevé (près d'un tiers du temps dans cinq séquences et entre $50 \%$ et $75 \%$ du temps dans quatre autres séquences $)^{10}$. Cela qui signifie que, par exemple, pour une séquence d'une durée moyenne de six heures, si un tiers du temps présente des ruptures de l'action didactique en raison d'élèves turbulents, de corrections de devoirs qui ne sont pas en lien avec la séquence (par exemple, corriger en grand groupe les pages d'un cahier d'exercices de grammaire) ou de problèmes avec le matériel informatique, c'est quatre heures d'enseignement de la poésie que les élèves auront véritablement reçues cette année-là.

L'écriture de poèmes est surtout travaillée au $1^{\mathrm{er}}$ cycle et est quasiment absente au $2^{\mathrm{e}}$ cycle, exception faite du projet haïku réalisé par Queenie, ce qui s'explique peut-être par la brièveté de ce genre d'écrit et par le jeu de contraintes ludiques qu'il sollicite en raison de sa structure. En revanche, la transmission de savoirs déclaratifs et la lecture, le plus souvent analytique d'ailleurs, se retrouvent dans la majorité des séquences. Quant à l'oral, aucun enseignant n'a demandé aux élèves de produire une performance poétique ; en revanche, quelques-uns leur ont fait écouter des chansons ou des poèmes oralisés avant de les analyser.

\section{Trames prototypiques : organisation d'ensemble et entrées des séquences}

Rappelons, à l'instar de J. Dolz et R. Gagnon (2018, p. 106), que les trames prototypiques représentent les suites d'activités qui présentent les savoirs dans le même ordre.

\section{Mouvement linéaire traditionnel dominant dans 8 des 10 séquences analysées}

Parmi les 10 séquences analysées, soulignons que ces séquences d'enseignement se terminent par une évaluation (nous y reviendrons), même si, selon les prescriptions officielles, il n'y a aucune obligation à évaluer de façon sommative la poésie. Cela dit, parmi ces 10 séquences, huit d'entre elles suivent le même mouvement linéaire comme l'illustre la figure ${ }^{11}$ suivante : 


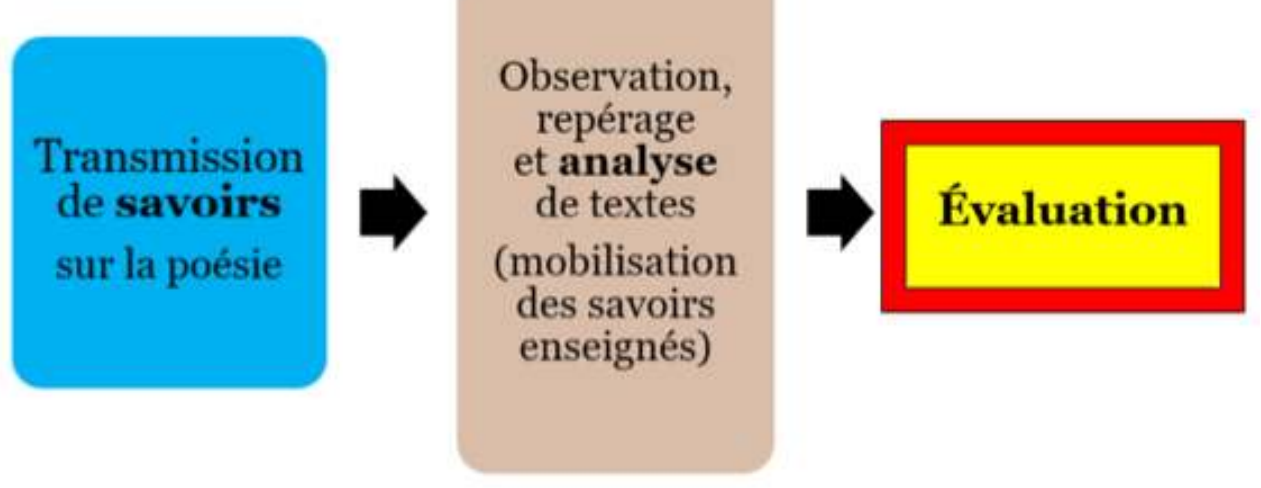

Après une première phase plus ou moins longue au cours de laquelle l'enseignante présente des savoirs déclaratifs sur la poésie de façon magistrale (par exemple: une longue liste de figures de style, chacune accompagnée de leur définition et d'une illustration), suit une phase de modelage, de mise en pratique ou d'exercisation à partir de textes poétiques lus ou entendus. Lors de cette deuxième phase pendant laquelle les élèves observent les textes, on leur demande d'abord de repérer les savoirs enseignés (par exemple: relever les figures de style dans un poème, identifier les mots qui constituent un champ lexical), puis de décomposer les textes en fonction de ces savoirs (par exemple : compter le nombre de syllabes, nommer les types de rimes) et, enfin, définir ces savoirs et leurs fonctions (par exemple: l'oxymore est une opposition et, dans ce poème, on illustre les tensions entre la mort et la vie).

\section{Deux cas qui se distinguent par une entrée se rapprochant de l'expérience poétique}

Deux séquences se distinguent de celles composées d'une trame prototypique plutôt traditionnelle: celles de Diane et de Tina, qui ont toutes les deux organisé leur séquence en commençant par une phase proche de l'expérience poétique, de façon à plonger leurs élèves dans l'univers poétique, à faire état de leurs représentations de la poésie en leur demandant d'écrire un court poème ou en suscitant leurs réactions et réflexions sur quelques textes lus ou entendus. 
Figure 2 : expérimenter pour apprendre (le cas de Diane)

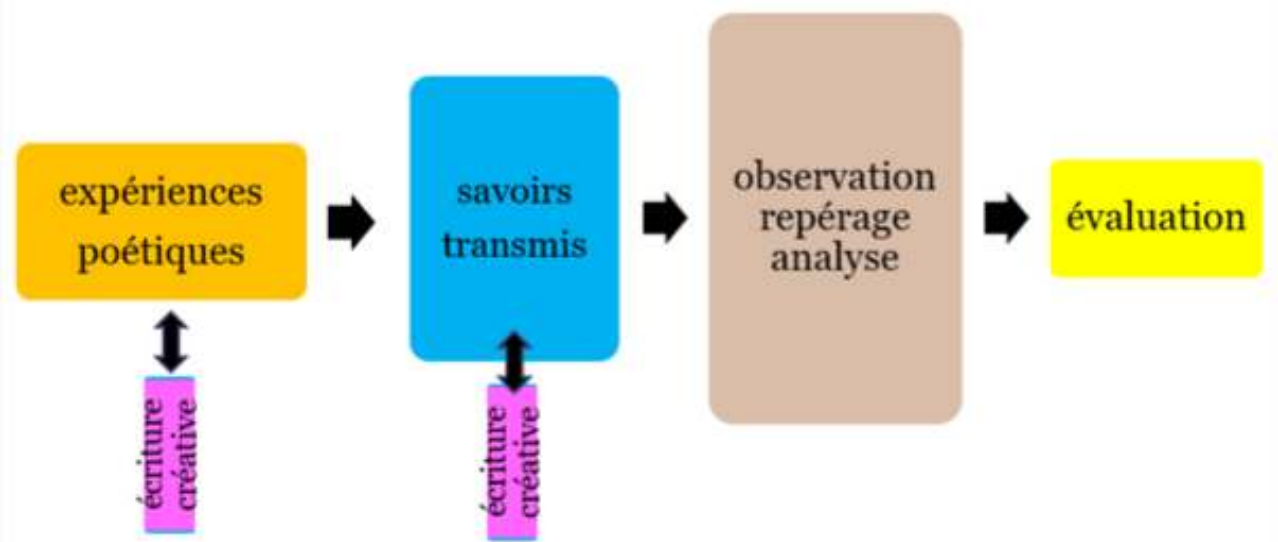

Dans sa séquence, Diane (voir figure 2) insère des activités d'écriture créative en lien avec les premières phases de sa séquence. Cela dit, ces courtes activités d'écriture ne représentent que $2,5 \%$ du temps total de sa séquence, soit environ 17 minutes d'une séquence de près d'11h30. Durée certes peu significative pour transformer le rapport à la poésie de ses élèves, il s'agit néanmoins d'une brèche qui ouvre la voie à l'expérience poétique. Par ailleurs, elle consacre le tiers du temps de sa séquence à la lecture et à l'analyse de 16 textes poétiques (il s'agit de l'une des trois séquences, sur les 10 analysées, dans lesquelles le temps de travail en lecture est le plus élevé). La première activité d'écriture créative n'était pas marquée de fortes contraintes d'écriture; elle a demandé à ses élèves d'écrire un court poème d'amour afin de faire émerger leurs représentations initiales de la poésie (par exemple : poésie $=$ rimes, comme amour + toujours), puis d'échanger sur leur production afin de générer des réactions des pairs. La deuxième activité fut de l'écriture automatique, pour amener les élèves à observer leur création et, à partir de leur propre texte, à repérer des savoirs enseignés (absence ou présence de rimes dans leur texte, identification de figures de style qu'ils ont inséré spontanément dans leurs écrits, etc.). Son objectif était de montrer aux élèves que la poésie n'est pas forcément un exercice de style formel, calculé ou mécanique; même par l'écriture automatique, et donc spontanée, il est possible de produire des figures de style ou des effets stylistiques que l'on considère poétiques, selon la représentation que l'on peut s'en faire.

Figure 3 : une progression spiralaire (le cas de Tina)

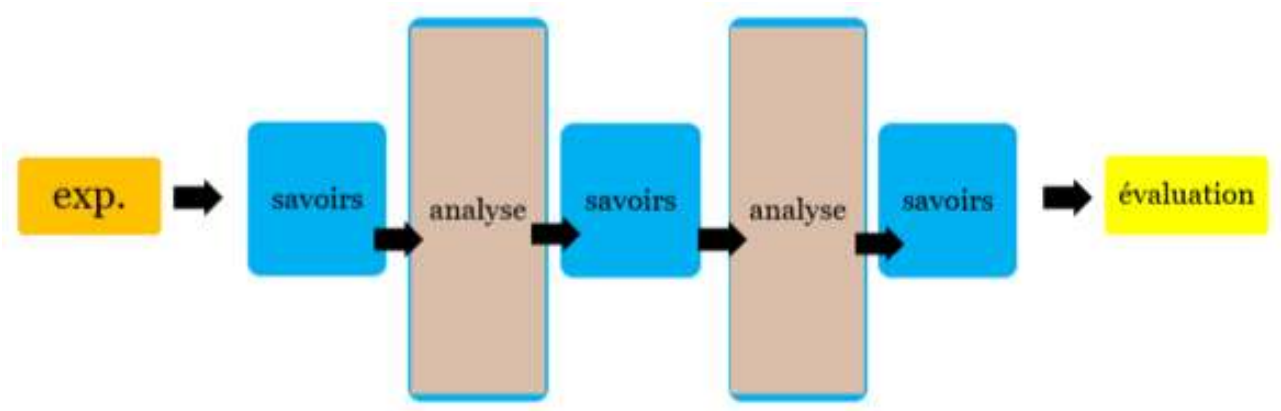

Tina est la seule des 10 enseignantes observées dont la séquence n'était pas organisée de façon linéaire, mais plutôt spiralaire (voir figure 3). En effet, elle faisait systématiquement des allers-retours entre quelques savoirs déclaratifs présentés aux 
élèves et observés dans quelques chansons tout en discutant en grand groupe des effets créés sur les réceptions singulières des élèves, puis elle transmettait d'autres savoirs poétiques et demandait de nouveau aux élèves d'écouter pour ensuite analyser d'autres chansons en mobilisant les premiers et deuxièmes savoirs enseignés, et ainsi de suite. Près de la moitié de sa séquence ( $46 \%$ du temps, soit environ 5 h 30 , sur une séquence de 12 heures) fut ainsi consacrée à la lecture participative ou analytique d'une trentaine de chansons et poèmes à l'aide de ces savoirs transmis progressivement.

\section{Sorties des séquences : un prolongement systématique vers l'évaluation sommative}

29 Comme nous l'avons souligné plus haut, toutes les séquences se terminent par une évaluation qui prend la forme d'un examen de lecture analytique ou d'une production écrite. Les examens de lecture des quatre enseignantes (Diane, Tina, Cindy, Cynthia) visent tous à amener les élèves à analyser un ou des poèmes en mobilisant les savoirs techniques enseignés ou en appliquant une grille/méthode d'analyse organisée à partir de ces savoirs. Diane soulignait d'ailleurs lors de l'entretien à quel point son travail est, bien malgré elle, de plus en plus conditionné par l'évaluation: "La formule qu'on enseigne [et] qu'on évalue [...] est: “je te montre une façon d'écrire" [que tu dois reproduire] ».

Quant aux productions écrites, trois types de tâches reviennent à la fin des séquences, soit 1) écrire à la manière de... en reproduisant les caractéristiques du genre étudié (Pathy, Denise, Queenie, Quiana); 2) écrire des poèmes créatifs sans imposer de contraintes spécifiques (Pauline, Tessa) ; 3) écrire un slam (Denise, Queenie).

Dans la majorité des cas, ce sont des évaluations traditionnelles, rendant opérationnelle l'évaluation de savoirs déclaratifs : par exemple dans un examen de lecture, identifier des figures de style dans un poème ou compter les syllabes dans les vers; dans une production écrite, écrire un haïku en respectant sa forme fixe... Car comment pourraiton mesurer l'expérience poétique, l'évènement de lecture ou évaluer un "poème du lecteur ", au sens de M. Favriaud (2011), à l'aide de critères observables et objectifs ? Les pratiques traditionnelles comme le repérage des figures de style et l'écriture d'imitation ou à contrainte servent l'évaluation, car elles offrent des critères objectifs pour juger les capacités des élèves. Par exemple, y a-t-il toutes les caractéristiques du haïku dans la production de l'élève? Retrouve-t-on dans le texte de l'élève les trois figures de style exigées, un champ lexical sur le thème choisi, la forme du sonnet, les alexandrins et les rimes croisées? L'élève a-t-il été en mesure de repérer les huit figures de style dans ce poème et de les nommer? Or ces pièges de l'évaluation conduisent à se demander si ce qui est évalué par les enseignants est davantage tourné vers les capacités des élèves à imiter, reproduire ou repérer, plutôt que celles d'interpréter, de créer ou de performer poétiquement, soit des pratiques qui se rapprochent davantage d'expériences poétiques telles qu'elles se vivent réellement, dans un rapport plus personnel et social, que scolaire et disciplinant. Lors des entretiens, plusieurs enseignants interrogés ont souligné que ces évaluations représentaient des freins à leur enseignement de la poésie, mais qu'ils étaient contraints de cumuler des notes pour les bulletins à venir ; cette contrainte scolaire, en plus des programmes chargés et du manque de temps que les enseignants dénoncent (Émery-Bruneau, 2018a), ne leur permet pas d'enseigner un objet sans qu'il ne devienne 
d'emblée un contenu évalué. Faire vivre une diversité d'expériences poétiques aux élèves sans en mesurer les effets sur leur apprentissage semble un luxe que la forme scolaire actuelle n'est pas prête à se payer.

\section{Savoirs enseignés explicitement et mobilisés pour analyser des poèmes}

Pour dégager l'élémentarisation des savoirs enseignés, nous avons analysé les niveaux 3 et 4 de nos synopsis (la synthèse des résultats de trouve aux annexes 1 et 2 ).

Du côté des savoirs enseignés sous forme d'exposé magistral, la majorité des enseignantes $(n=7 / 10)$ présentent de longues listes de figures de style (nom, définition, exemple) et la moitié d'entre elles $(n=5 / 10)$ illustrent les structures des poèmes à formes fixes (par exemple : quatrains, tercets et alexandrins qui composent le sonnet). Dans une moindre mesure, les savoirs suivants reviennent aussi de façon systématique : règles de versification, champs lexicaux pour illustrer un thème, contexte de production d'un poème (courant littéraire ou contexte sociohistorique).

Quant aux savoirs que les enseignantes demandent aux élèves de mobiliser pour analyser les poèmes, c'est une méthode de lecture analytique qui est le plus souvent sollicitée $(n=7 / 10)$, en plus du repérage de figures de style dans un poème $(n=5 / 10)$, et le tiers des enseignantes demande d'identifier des formes ou structures des poèmes, d'analyser la versification (scander et compter le nombre de pieds ou nommer le type de rimes), de relever les champs lexicaux afin de faire émerger le thème dominant et de donner son avis sur le poème (appréciation de l'élève). La méthode de lecture analytique est principalement enseignée dans le but de les préparer à l'examen dans lequel ils devront de nouveau la reproduire, ce qui se rapproche de la pratique pédagogique "teaching to the test» (Popham, 2001) tournée vers des finalités de rentabilité du temps d'apprentissage par des exercices ciblés et de performance des élèves qui se sont entrainés dans l'unique but de réussir l'examen. La méthode d'analyse de Diane, qu'elle présente en sept étapes dans la troisième phase de sa séquence (celle sur l'observation et l'analyse de poèmes), en est un exemple représentatif : «1) imager le texte, 2) nommer la forme du poème, 3) cerner le mode de discours du poème (narratif, descriptif, argumentatif), 4) identifier les thèmes en encerclant les champs lexicaux, 5) nommer les rimes (plates, embrassées, croisées), 6) nommer le registre de langue et le justifier (preuves), 7) nommer les figures de style repérées ». De telles méthodes d'analyse, qui demeurent répandues dans les pratiques d'enseignement de la poésie, sont davantage tournées vers un travail de repérage d'éléments, à l'aide de savoirs mobilisés, que vers l'analyse pour construire la compréhension et l'interprétation, exception faite de la première étape (imager le texte = stratégie de lecture, soit illustrer pour mieux se représenter le discours) et de la quatrième étape (inférer des thèmes pour construire le sens du poème). Cette méthode très scolaire facilite le travail d'évaluation, tout en évacuant d'emblée toute possibilité au sujet lecteur de s'exprimer sur sa réception singulière du poème ou d'expliciter ses interprétations, ses réflexions, ce qui a fait évènement pendant sa lecture ou ses jugements critiques sur le texte. 


\section{Noyau dur de l'enseignement de la poésie}

Par noyau dur, nous entendons un noyau notionnel qui organise l'enseignement et l'apprentissage d'un objet, qui est relativement stable et répétitif, quelle que soit l'approche mise en œuvre ou même le degré/cycle où il est convoqué. De fortes tendances se dégagent de nos 10 synopsis. Du côté des pratiques langagières, l'enseignement de la poésie est marqué, d'une part, par l'écriture d'imitation ou de reproduction, et de l'autre, par la lecture méthodique et analytique guidée par une grille de lecture, soit un outil constitué de savoirs scolaires mobilisés. Quant aux éléments de contenus, ce qui renvoie à l'élémentarisation de l'objet, ce noyau dur est composé de quatre savoirs fondamentaux qui reviennent dans la majorité des cas et qui renvoient tous à des critères internes aux textes littéraires : figures de style, formes et structure des textes, règles de versification, champs lexicaux et thèmes.

Ce noyau dur de l'enseignement de la poésie est marqué de transpositions didactiques plus ou moins heureuses provenant de la poétique, du formalisme, du structuralisme et de la linguistique. Comme si les enseignants partaient du postulat que la poésie est «naturellement réputée illisible» (Balpe, 1980) et qu'on ne peut en faire émerger des interprétations diverses, car lire de la poésie consisterait essentiellement à « analyser ce que le poème dit » (Joubert, 2015 [1977], p. 197) et non ce que le poème me dit. Ainsi, en plus d'observer l'absence d'activités pour travailler la production orale en poésie (par exemple: poésie sonore, performance slamée), le travail d'interprétation et d'appréciation des lecteurs est peu présent dans les pratiques d'enseignement, évacuant d'emblée le "poème du lecteur ", au sens de M. Favriaud (2011). En misant essentiellement sur un travail d'analyse méthodique ou de reproduction de caractéristiques spécifiques à un genre poétique, la place du « sujet » est peu présente. On occulte ainsi la manière dont la poésie fait évènement en lui, l'amène à vivre des expériences sensorielles, langagières ou existentielles variées, sollicite des réflexions profondes sur le sens et les significations à donner au poème - et plus largement à la poésie - au point d'en transformer son rapport au langage, à lui-même et au monde.

\section{Transformations de l'enseignement de la poésie au secondaire québécois?}

À l'heure où la poésie contemporaine est des plus diversifiée (prose ou vers libre, absence de rimes, utilisation du numérique, éditions alternatives, performances...), nos résultats montrent que l'école contribue encore à nourrir le mythe d'une poésie classique nécessitant des outils savants pour en dégager un sens inclus dans le texte, lequel étant plus ou moins construit en articulation avec la réception des lecteurs. D'ailleurs, certaines pratiques se sont cristallisées à travers l'histoire de notre discipline (Chervel, 2006) et arrivent toujours en tête des pratiques d'enseignement de la poésie au secondaire : l'écriture d'imitation et la lecture analytique. En revanche, la récitation et les dissertations ont disparu des pratiques au secondaire québécois; la seconde est néanmoins toujours présente dans tous les cours de littérature obligatoires du postsecondaire (Cégep; Babin, 2016). Puis, de nouvelles couches de sédimentation des pratiques ont pris forme: on ne demande plus aux élèves d'imiter le style des auteurs, mais plutôt les caractéristiques des genres formels qu'ils étudient. On ne leur demande plus de réciter ou d'ânonner un poème, mais plutôt d'écouter une chanson ou 
de slamer. Dans quelques rares cas, on invite les élèves à vivre des expériences poétiques, puis à exprimer leurs réactions et leurs appréciations des poèmes lus ou entendus : l'émergente de cette pratique dans les classes s'explique entre autres par la compétence "Lire et apprécier des textes littéraires » (Ministère de l'Éducation du Québec, 2005 ; Ministère de l'Éducation, du Loisir et du Sport du Québec, 2009). Ainsi, depuis une quinzaine d'années, les prescriptions invitent à engager davantage les élèves dans une démarche axiologique et réflexive lorsqu'ils se retrouvent devant des textes littéraires de tout genre.

\section{Conclusion : pour une réflexion didactique et créatrice}

De cette recherche, une question fondamentale est demeurée au cœur de notre travail : quels lecteurs de poésie forme-t-on à travers les pratiques d'enseignement de cet objet? Et la sous-question qui s'en est suivie est: à partir de ce qui se fait présentement, et en tenant compte des conditions et contextes de travail des enseignants du secondaire, quels lecteurs de poésie peut-on former?

K. Canvat et G. Legros $(1997$, p. 26) militaient il y a près de 25 ans pour défendre un "véritable enseignement de la poésie [qui] devrait viser à former des lecteurs connaisseurs et pas seulement des amateurs " (nous soulignons). En 2004, après avoir analysé les discours des didacticiens de français, tirés des revues scientifiques publiées entre 1970 et 2003, S. Richard montre qu'il existe une finalité "fondamentale » de l'enseignement de la littérature et de la lecture de textes littéraires au secondaire. Celle-ci vise à former un lecteur lettré, soit "non seulement un amateur de littérature, mais également un connaisseur qui peut participer au monde littéraire en commentant, en critiquant et en interprétant les œuvres littéraires " (Richard, 2004, p. 2). Cette tension entre connaissances et appétence a encore été soulevée récemment par M. Lépine (2017) qui défend, quant à lui, l'importance de former des «amateurs éclairés ", au sens de J.-L. Dumortier (2001), soit des lecteurs qui développeront le gout de lire et sauront apprécier des œuvres littéraires, afin qu'ils deviennent non seulement des lecteurs appétents, mais également compétents.

Or, à la lumière de nos résultats, nous pouvons nous demander: mais où sont les lecteurs amateurs et appétents à l'école secondaire, du moins lorsque l'on enseigne la poésie? Car rappelons-le, les trames prototypiques que nous avons dégagées et le noyau dur qui constitue les pratiques ont pour principales finalités de transmettre des savoirs poétiques, formels et linguistiques, afin de donner des outils surtout normatifs pour « décrypter » des poèmes. Ce lecteur « amateur » ou " appétent » qui s'autorise à convoquer sa subjectivité pour vivre des lectures créatives et à manifester sa réflexivité lorsqu'il en rend compte à d'autres lecteurs existe-il vraiment en classe ? Pourtant, lors des entretiens menés avec les enseignants participant à notre recherche (ÉmeryBruneau, 2018a), plusieurs d'entre eux ont révélé avoir écrit de la poésie pendant leur adolescence, dans leur temps libre, et que cela représentait une pratique poétique personnelle positive pour eux (l'histoire de la poésie n'est-elle pas par ailleurs marquée de poètes adolescents célèbres comme A. Rimbaud et É. Nelligan ?). Ce lecteur amateur ou appétent peut-il même exister en classe ? Comme didacticiens, devrions-nous faire le deuil d'un enseignement de la poésie tourné vers la lecture-expérience, qui ne peut prendre place que dans la vie en dehors de l'école, une fois que l'école aura fourni les savoirs et outils techniques qui permettront éventuellement de parvenir à cet idéal ? 
Nos résultats montrent que l'espace accordé à ce sujet-lecteur demeure encore marginal dans les pratiques d'enseignement et d'évaluation actuelles. En outre, malgré ces principes didactiques défendus depuis les années 1970 et repris par plusieurs didacticiens au fil des décennies, arrivons-nous vraiment à former des lecteurs connaisseurs si, nonobstant les cycles/degrés d'enseignement, nous constatons qu'il n'y a qu'un même noyau dur des savoirs déclaratifs en poésie qui constitue le cœur de cet objet et qui est (re)transmis et (ré)enseigné à tous les degrés au fil de la scolarité secondaire et par la suite collégiale (cégep), bien souvent?

41 Nous nous demandons également quelle est la place pour l'expérience poétique et l'avènement du sujet-lecteur à l'école. Et cette réflexion va bien au-delà des critères qui en rendraient objectivable son évaluation. En effet, nous observons une rupture entre, d'un côté, les pratiques scolaires, et de l'autre, les pratiques sociales de référence en poésie contemporaine ou même les pratiques encouragées par les didacticiens de la poésie pour articuler les savoirs aux pratiques langagières du dire-lire-écrire (Favriaud, Vinsonneau \& Poletto, 2017), dont, à tire d'exemples, le recueil à quatre mains ou l'annotation à plusieurs dans les marges le texte pour le marquer de ses réactions ou interprétations (Brillant-Rannou, 2016); les performances poétiques pour articuler mise en voix et corps à un travail de compréhension et d'interprétation des poèmes (Émery-Bruneau \& Brunel, 2016; Martin, 2018b); ou pour analyser les effets du numérique sur la poésie (Brillant-Rannou, Boutevin, \& Brunel, 2016 ; Massol, 2018). Du reste, comme l'ont montré A. Rouxel et S. Lemarchand (2018), ce sont les modèles réglés ou conformes aux modèles scolaires qui appellent des remarques formelles. Donc, comment arriver à mettre en place un enseignement de la poésie qui aura pour objectif que "les lectures réelles deviennent productives d'effets et de sens non programmés par l'auteur... ou le professeur [...] [car] diverses expérimentations témoignent du fait que rien ne devrait s'y opposer, une fois la formation et la confiance des enseignants assurées " (Brillant-Rannou, 2018, p. 255-256) ? Comment créer cette confiance sinon, d'abord, en explorant avec eux leur rapport personnel à la poésie et en les accompagnant au fil des évènements de lecture qu'ils vivront, qui les bousculeront et, peut-être, les transformeront ? Comment gagner le pari d'un enseignement de la poésie qui se rapproche à la fois des pratiques sociales de référence en poésie et des pratiques de lecture réelles, marquées d'évènements qui donnent forme à la motricité des lectures créatives, car "si le poème est évènement, c'est parce que cela parle de nouveau, cela lie, cela ouvre et cela restitue à soi » (Martinez, 2018, p. 228) ? Mais peutêtre qu'une partie de la réponse réside dans la question. Et si on répartissait la formation en poésie et, plus largement, en littérature entre, d'une part, des savoirs sociaux, techniques et historiques étudiés en classe, bien que peu didactisés, comme nous l'observons dans les pratiques sédimentées actuelles, et d'autre part, demandions aux élèves et aux enseignants de vivre des expériences poétiques réelles en dehors de la classe, en assistant à des performances poétiques, à des soirées de poésie, à des joutes de slam, à un festival international de poésie ou à un bingo poétique, par exemple ${ }^{12}$ ? La rencontre entre ces deux espaces de formation s'incarnerait ensuite en classe, au moment de rendre compte de ce qui a fait évènement, de ce qui les a émus, de ce qui a transformé le rapport à la poésie de ces sujets : les savoirs sur la poésie seront les outils et le métalangage qui leur permettront d'analyser leurs expériences poétiques et d'en rendre compte.

Si les enseignants demeurent pour la plupart dans des pratiques d'enseignement traditionnelles, c'est peut-être parce que la formation qu'ils ont reçue reste insuffisante 
pour transformer leur manière de travailler la poésie en classe. Partant, pour former des enseignants amateurs de poésie, qui se sentiront plus à l'aise de l'enseigner, et connaisseurs, pour accompagner leurs élèves au fil de leurs expériences poétiques, la formation initiale et continue des enseignants doit manifestement être améliorée. En effet, dans les programmes de formation des enseignants de français du secondaire, offerts parmi les neuf universités francophones québécoises, seulement trois de ces neuf programmes proposent un cours disciplinaire obligatoire qui porte spécifiquement sur la poésie ${ }^{13}$ et deux autres programmes offrent un cours où la poésie est l'un des genres étudiés parmi d'autres ${ }^{14}$. En outre, aucune université n'offre de cours de didactique axé spécifiquement sur la poésie, cette dernière étant néanmoins évoquée dans quelques descriptifs des cours de didactique de la littérature, de la lecture littéraire ou de la création littéraire, ce qui signifie qu'elle peut être étudiée dans une perspective didactique, ou non, selon les choix des formateurs.

Alors, compte tenu de ce qui précède, on doit se demander quelles peuvent être les retombées concrètes des recherches récentes en didactique de la poésie sur la formation des enseignants et les pratiques enseignantes. Demeurer sur le terrain des savoirs savants nous semble limitatif; et si nous transformions nos cours en y intégrant une portion de la formation qui serait de type «stage culturel » pour former les futurs enseignants à vivre des expériences poétiques dans les milieux littéraires et culturels de leur région, afin qu'ils puissent ensuite élaborer différentes activités d'enseignement et d'apprentissage qui leur permettraient de solliciter de telles expériences chez leurs futurs - élèves. M. Lépine et A. Bélanger (2021, Sous presse) ont expérimenté une telle approche dans le cadre d'un projet-pilote qui articule éducation informelle et formelle pour mieux former les passeurs culturels, et ce projet présente des effets certains sur la transformation de la culture seconde des futurs enseignants, à condition qu'ils s'engagent avec détermination à vouloir s'émanciper, notamment par la culture et la littérature.

Quant à la formation continue, non obligatoire et rarissime pour les enseignants du Québec, si l'absence de volonté politique demeure pour dégager du temps dans la tâche des enseignants afin qu'ils puissent bonifier sur une base hebdomadaire leur formation tout au long de leur carrière, nous devons, comme chercheurs, trouver d'autres moyens de faire circuler nos savoirs. Pour ce faire, divers types de recherches peuvent certes être mises en place, notamment l'ingénierie didactique collaborative, les recherches développement, les recherches action, etc., bref des recherches menées en collaboration avec les enseignants, en fonction de leur contexte d'enseignement. En mettant en place de telles recherches, nous pourrions, par exemple, miser sur les activités d'entrées dans une séquence pour réfléchir avec eux aux espaces à donner aux expériences poétiques et aux évènements de lecture, à la manière d'en susciter, au corpus à utiliser pour y arriver, etc. Ces types de recherche, bien que stimulantes et engageant directement les enseignants dans les décisions, présentent néanmoins plusieurs limites, à commencer par le nombre de participants très limités qui peuvent $\mathrm{y}$ prendre part en raison du temps nécessaire à y investir et des couts élevés pour financer ce type de recherche. Une autre option serait la mise en place de communautés d'apprentissage professionnelles (CAP) qui pourraient être orientées vers quelques pratiques sociales et culturelles auxquelles les enseignants participeraient (par exemple, tous les enseignants de cette CAP assisteraient ensemble à une joute de slam), puis ils objectiveraient ensuite leurs expériences poétiques lors des discussions et débats au sein de leur CAP qui ont lieu pendant leurs heures de 
travail rémunéré. Ainsi s'engageraient-ils volontairement à transformer leur culture première et à réfléchir ensemble aux retombées de telles transformations personnelles sur leurs pratiques d'enseignement.

Entre appétence, connaissances et expériences, il est peut-être temps de penser à une didactique créatrice d'espaces à investir, créatrice d'expériences, plutôt qu'essentiellement créatrice de connaissances. Il s'agit du moins de quelques chantiers à explorer pour permettre aux enseignants de renouer avec la poésie et son enseignement en renouvelant leurs pratiques qui reposent encore sur des approches issues de la linguistique et du formalisme, et pour les amener à faire vivre à leurs élèves de véritables expériences poétiques.

\section{BIBLIOGRAPHIE}

AEBY DAGHÉ, S. (2014). Candide, la fée carabine et les autres. Vers un modèle didactique de la lecture littéraire. Bruxelles/Berne/Berlin : P. Lang. En ligne : https://www.peterlang.com/view/title/ 36164.

BABIN, J. (2016). La lecture d'œuvres littéraires complètes au collégial : des cas de pratiques d'enseignement dans le cours d'Écriture et Littérature. Thèse de doctorat en éducation : Université de Sherbrooke. En ligne : https://savoirs.usherbrooke.ca/bitstream/handle/11143/9782/

Babin_Julie_PhD_2016.pdf?sequence=1\&isAllowed=y.

BALPE, J.-P. (1980). Lire la poésie. Paris : A. Colin/Bourrelier.

Baudelot, C., CARTier, M. \& Detrez, C. (1999). Et pourtant, ils lisent... Paris : Éd. du Seuil.

BRILLANT-RANNOU, N. (2010). Le Lecteur et son poème. Lire en Poésie : expérience littéraire et enjeux pour l'enseignement $d u$ français en lycée. Thèse de doctorat en littérature française : Université Rennes 2. En ligne : https://www.bu.univ-rennes2.fr/sites/default/files/theses/These_NR_HAL.pdf.

BRILLANT-RANNOU, N. (2016). « Le recueil à quatre mains et la lecture dialoguée dans les marges : conception et expérimentation de deux dispositifs de lecture subjective de poésie ». In : BrillantRannou, N., Boutevin, C. \& Brunel, M. (dirs). Être et devenir lecteur(s) de poèmes. De la poésie patrimoniale au numérique. Namur : Presses universitaires de Namur, p. 87-109.

BRILlANT-RANNOU, N. (2018). « Horizons ». In : Boutevin, C., Brillant Rannou, N. \& Plissoneau, G. (dir). À l'écoute des poèmes. Enseigner des lectures créatives. Bruxelles : P. Lang, p. 255-257.

BRILLANT-RANNOU, N. \& BOUTEVIN, C. (2018). «Vers des réceptions créatives en poésie. Origines et mesures de la question ». In : Boutevin, C., Brillant Rannou, N. \& Plissoneau, G. (dir). À l'écoute des poèmes. Enseigner des lectures créatives. Bruxelles : P. Lang, p. 11-20.

BRILLANT-RANNOU, N. \& PETIT, C. (2015). « Devenir lecteur et scripteur de poésie en primaire : quelle expérience ? Quels enjeux? ». Repères 52, p. 159-176. En ligne : https://journals.openedition.org/ reperes/967.

BRILLANT-RANNOU, N., BOUTEVIN, C. \& BRUNEL, M. (dirs) (2016). Être et devenir lecteur(s) de poèmes. De la poésie patrimoniale au numérique. Namur : Presses universitaires de Namur. 
BERGERON, H. (2016). La Poésie à l'école secondaire québécoise (1963-2016). Une histoire à travers les textes. Thèse de doctorat en études littéraires : Université Laval. En ligne : https://corpus.ulaval.ca/ jspui/bitstream/20.500.11794/27280/1/32723.pdf.

CANVAT, K. \& LEgros, G. (1997). « Enseigner la poésie moderne ? ». Pratiques 93, p. 5-29. En ligne : https://www.persee.fr/doc/prati_0338-2389_1997_num_93_1_1792.

CHERVEL, A. (2006). Histoire de l'enseignement $d u$ français $d u X V I I^{e}$ au XXe siècle. Paris : Retz.

DOLZ, J. \& GAGNON, R. (dirs) (2018). Former à enseigner la production écrite. Villeneuve d'Asqc : Presses universitaires du Septentrion.

DUFAYS, J.-L., LISSE, M. \& MEURÉE, C. (2009). Théorie de la littérature. Une introduction. Louvain-laNeuve : Academia Bruylant.

DUMORTIER, J.-L. (2001). Lire le récit de fiction. Pour étayer un apprentissage : théorie et pratique. Louvain-la-Neuve : De Boeck/Duculot.

DUVAL, I. (2004). « La poésie et l'enseignement du français ». Québec français135, p. 42-45. En ligne : https://www.erudit.org/en/journals/qf/2004-n135-qf1183720/55545ac.pdf.

ÉMERY-BRUNEAU, J. (2018a). « Le rapport à la poésie d'enseignants du secondaire québécois : quelle progression entre les cycles/degrés? ». Trema. Revue internationale en science de l'éducation et didactique 49, p. 29-42. En ligne : https://journals.openedition.org/trema/4526.

ÉMERY-BRUNEAU, J. (2018b). « Finalités de l'enseignement de la poésie au secondaire québécois ». Pratiques 179-180. En ligne : https://journals.openedition.org/pratiques/4747.

ÉMERY-BRUNEAU, J. \& BRUNEL, M. (2016). « Poésie oralisée et performée : quel objet, quels savoirs, quels enseignements? ». Repères. Recherches en didactique du français langue maternelle 54, p. 189-206. En ligne : https://journals.openedition.org/reperes/1117.

FAVRIAUD, M. (2011) « Le poème du lecteur : mémorisation, imagination, compréhension - et image de soi ». In : Mazauric, C., Fourtanier, M.-J. \& Langlade, G. (dirs). Le Texte du lecteur. Bruxelles : P. Lang, p. 175-192.

FAVRIAUD, M., (2018). « L'entretien didactique en écopoésie au cycle 2 de l'école primaire. Entre interprétation dérivante et réflexivité ». In : Boutevin, C., Brillant Rannou, N. \& Plissoneau, G. (dir). À l'écoute des poèmes. Enseigner des lectures créatives, Bruxelles : P. Lang, p. 77-97.

FAVRiaud, M., VInSONNEAU, M. \& POLETTO, M. (2017). Les Chemins de poésie d'Alep. Poétique et didactique du dire-lire-écrire à l'école primaire. Limoges : Lambert Lucas.

JOUBERT, J.-L. (2015) [1977]. La Poésie. Paris : A. Colin.

LÉPINE, M. (2017). L'Enseignement de la lecture/appréciation des œuvres littéraires à l'école primaire : enquête sur les pratiques déclarées et les conceptions d'enseignants québécois. Thèse de doctorat en science de l'éducation. Université de Montréal. En ligne : https://papyrus.bib.umontreal.ca/ $\mathrm{xmlui} /$ bitstream/handle/1866/20052/Lepine_Martin_2017_these.pdf?sequence=3\&isAllowed=y.

LÉPINE, M. \& BÉLANGER, A. (2021, Sous presse). « Former des Passeurs culturels dès la formation initiale en enseignement ou comment mieux articuler éducation informelle et formelle en matière de culture? ». In : Maulini, O. et al. (dirs). La Formation buissonnière des enseignants. De leurs apprentissages informels à ce que leurs études en font. Louvain-La-Neuve : De Boeck.

LOUicHon, B. (2011). « La lecture littéraire est-elle un concept didactique? ». In : Reuter, Y. et al. (dirs). Les Concepts et les méthodes en didactique du français, Belgique : Presses universitaires de Namur, p. 195-216. 
MASSART-LALUC, V. (2010). « De la difficulté à enseigner et évaluer la poésie ». Lire au lycée professionnel 62 (3). En ligne : http://www.educ-revues.fr/LLP/AffichageDocument.aspx? iddoc $=38879$.

MARTIN, S. (2018a). « Un petit caillou dans la chaussure didactique : le poème ». Pratiques 179-180. En ligne : http://journals.openedition.org/pratiques/4710.

MARTIN, S. (2018b). « Le poème, relation de voix : une activité critique ». In : Boutevin, C., BrillantRannou, N. \& Plissoneau, G. (dirs). À l'écoute des poèmes. Enseigner des lectures créatives. Bruxelles : P. Lang, p. 23-39.

MARTINEZ, V. (2018). «Que faire de la notion d'événement en didactique du poème ?». In :

Boutevin, C., Brillant-Rannou, N. \& Plissoneau, G. (dirs). À l'écoute des poèmes. Enseigner des lectures créatives. Bruxelles : P. Lang, p. 225-238.

MASSOL, J.-F. (2016). « Lire subjectivement un sonnet de Paul Verlaine en classe de secondaire : variété, difficultés et intérêts d'une démarche ». In : Brillant-Rannou, N., Boutevin, C. \& Brunel, M. (dirs), Être et devenir lecteur(s) de poèmes. De la poésie patrimoniale au numérique. Namur : Presses universitaires de Namur, p. 111-134.

MASSOL, J.-F. (2018). « Écouter/voir des poèmes à l'époque d'internet ». In : Boutevin, C., BrillantRannou, N. \& Plissoneau, G. (dirs). À l'écoute des poèmes. Enseigner des lectures créatives. Bruxelles : P. Lang, p. 181-203.

MAZAURIC, C., FOURTANiER, M.-J. \& LANGLADE, G. (dirs) (2011). Le Texte du lecteur. Bruxelles : P. Lang.

Ministère de l'Éducation du Québec (2005). Programme de formation de l'école québécoise.

Enseignement secondaire, premier cycle. Québec : Gouvernement du Québec. En ligne : http:// www.education.gouv.qc.ca/fileadmin/site_web/documents/PFEQ/prfrmsec1ercyclev2.pdf.

Ministère de l'Éducation, du Loisir et du Sport du Québec (2009). Programme de français, langue d'enseignement, du deuxième cycle du secondaire. Québec : Gouvernement du Québec. En ligne : http://www.education.gouv.qc.ca/fileadmin/site_web/documents/education/jeunes/pfeq/ PFEQ_francais-langue-enseignement-deuxieme-cycle-secondaire.pdf.

Ministère de l'Éducation, du Loisir et du Sport du Québec (2011). Progression des apprentissages au secondaire. Français, langue d'enseignement. Québec : Gouvernement du Québec. En ligne : http:// www.education.gouv.qc.ca/fileadmin/site_web/documents/education/jeunes/pfeq/ PDA_PFEQ_francais-langue-enseignement-secondaire_2011.pdf.

NELSEN, D. (1960). « Bill Evans ». Downbeat 27, p. 16.

POPHAM, W. J. (2001). « Teaching to the test ». Educational Leadership 58 (6), p. 16-20.

RICHARD, S. (2004). Finalités de l'enseignement de la littérature et de la lecture de textes littéraires au secondaire. Thèse de doctorat en didactique. Université Laval.

ROUXEL, A. \& LEMARCHAND, S. (2018). « À l'écoute des poèmes, entre fascination et résilience ». In : Boutevin, C., Brillant Rannou, N. \& Plissoneau, G. (dirs). À l'écoute des poèmes. Enseigner des lectures créatives, Bruxelles : P. Lang, p. 239-254.

SCHNEUWLY, B. \& DOLZ, J. (2009). Les Objets enseignés en classe de français. Rennes : Presses universitaires de Rennes.

THÉVENAZ-CHRISTEN, T. et al. (dirs) (2014). La Lecture enseignée au fil de l'école obligatoire. L'exemple genevois. Namur : Presses universitaires de Namur. 


\section{ANNEXES}

\section{Annexe 1}

Tableau synthèse des résultats (temps d'enseignement des savoirs déclaratifs transmis pendant les séquences)

\begin{tabular}{|c|c|c|c|c|c|c|c|c|c|c|c|}
\hline $\begin{array}{l}\text { SANOIRS ENSEIGNÉS EN POÉSIE } \\
\text { (niveaux } 3 \text { et 4) }\end{array}$ & $\begin{array}{l}\text { Pathy } \\
\text { (fable) }\end{array}$ & $\begin{array}{l}\text { Pauline } \\
\text { (poésie) }\end{array}$ & $\begin{array}{l}\text { Diane } \\
\text { (poésic) }\end{array}$ & $\begin{array}{l}\text { Denise } \\
\text { (slam) }\end{array}$ & $\begin{array}{l}\text { Tessa } \\
\text { (écriture) }\end{array}$ & $\begin{array}{c}\text { Tina } \\
\text { (chanson) }\end{array}$ & $\begin{array}{l}\text { Quiana } \\
\text { (haiku) }\end{array}$ & $\begin{array}{l}\text { Queenie } \\
\text { (slam) }\end{array}$ & $\begin{array}{c}\text { Cindy } \\
\text { (chanson) }\end{array}$ & $\begin{array}{c}\text { Crnthia } \\
\text { (engagé) }\end{array}$ & TOTAL \\
\hline Organisation du texte poétique & & & & & & & & & & & 16 \\
\hline forme (fixe ou libre), structure (quatrain, strophe) & $3 \mathrm{~m} 00$ & $6 \mathrm{~m} 40$ & $5 \mathrm{~m} 35$ & & & $13 \mathrm{~m} 45$ & $7 \mathrm{~m} 55$ & & & & 5 \\
\hline versification (vers, strophe, couplets, etc.) & & $2 \mathrm{m50}$ & & & & $8 \mathrm{~m} 25$ & & & $20 \mathrm{~m} 25$ & & 3 \\
\hline rythmes et sonorités (rimes, répétition, etc.) & & $5 \mathrm{~m} 15$ & & & & $4 \mathrm{~m} 10$ & & $1 \mathrm{~m} 05$ & $13 \mathrm{~m} 40$ & & \\
\hline genre et caractéristiques textuelles & $5 \mathrm{~m} 35$ & & & $3 \mathrm{~m} 45$ & & & $2 \mathrm{~m} 00$ & $4 \mathrm{~m} 50$ & & & 4 \\
\hline Univers poétique & & & & & & & & & & & \\
\hline sens, connotation, message & $4 \mathrm{~m} 15$ & $0 \mathrm{~m} 55$ & & & & $2 \mathrm{~m} 15$ & & & $2 \mathrm{~m} 45$ & & 3 \\
\hline thèmes et champs lexicaux & & $1 \mathrm{~m} 40$ & & $2 \mathrm{~m} 10$ & & $7 \mathrm{~m} 45$ & $3 \mathrm{~m} 10$ & & & & 4 \\
\hline vision du monde, intention, point de vue & & & & & & $2 \mathrm{~m} 15$ & $0 \mathrm{~m} 25$ & $0 \mathrm{~m} 30$ & & & 1 \\
\hline voix poétique & & & & & & $12 \mathrm{~m} 50$ & & & $1 \mathrm{mo}$ & & 2 \\
\hline éléments de l'univers poétique & $3 \mathrm{~m} 00$ & & & & & $2 \mathrm{~m} 20$ & & $3 \mathrm{~m} 20$ & & & 3 \\
\hline $\begin{array}{r}\text { Langue } \\
\end{array}$ & & & & & & & & & & & 14 \\
\hline vocabulaire/lexique & & & & & & $2 \mathrm{~m} 10$ & & & & & 1 \\
\hline figures de style, procédés d'écriture & $1 \mathrm{~m} 35$ & $10 \mathrm{~m} 35$ & $54 \mathrm{~m} 30$ & & & $38 \mathrm{~m} 15$ & & $8 \mathrm{~m} 40$ & $4 \mathrm{~m} 30$ & $20 \mathrm{~m} 45$ & 7 \\
\hline ponctuation & & & & & & $2 \mathrm{~m} 35$ & & & & & 1 \\
\hline variété/registre de l & & & & & & $1 \mathrm{~m} 30$ & & $0 \mathrm{~m} 25$ & $4 \mathrm{~m} 40$ & & 2 \\
\hline mode de discours & & & & & & $1 \mathrm{~m} 30$ & & & $2 \mathrm{~m} 05$ & $6 \mathrm{~m} 45$ & 3 \\
\hline Contexte de production d'une cuvre & & & & & & & & & & & \\
\hline biographie de Pauteur, genèse de son travail & $12 \mathrm{~m} 00$ & & & & & & $1 \mathrm{~m} 10$ & $1 \mathrm{~m} 35$ & & & 3 \\
\hline conteste social, historique, politique & $1 \mathrm{~m} 00$ & & & & & $6 \mathrm{~m} 10$ & $2 \mathrm{~m} 45$ & $3 \mathrm{~m} 25$ & & & 4 \\
\hline institution littéraire, courant littéraire ou artistique & & & & $3 \mathrm{~m} 50$ & & & $1 \mathrm{~m} 55$ & $4 \mathrm{~m} 40$ & & & 3 \\
\hline objet texte o & $2 \mathrm{~m} 35$ & & & & & & & & & & 1 \\
\hline Contexte de réception d'un texte & & & & & & & & & & & \\
\hline appréciation ou jugement & & & & & & & & $2 \mathrm{~m} 55$ & & & 1 \\
\hline interprétation ou compréhension & $4 \mathrm{~m} 20$ & & $12 \mathrm{~m} 10$ & & & & & $13 \mathrm{~m} 10$ & & & 3 \\
\hline & & & & & & & & & & & \\
\hline Nombre total de savoirs enseignés & 9 & 7 & 3 & 3 & 0 & 14 & 7 & 11 & 8 & 2 & \\
\hline $\begin{array}{l}\text { TOTAL de temps consacré dans la séquence } \\
\text { pour transmettre des notions poétiques }\end{array}$ & $\begin{array}{l}\mathrm{h} 15 \mathrm{~m} 15 \\
16,7 \%\end{array}$ & $\begin{array}{l}18 \mathrm{~m} 25 \\
6 \%\end{array}$ & $\begin{array}{l}1 \mathrm{~h} 28 \mathrm{~m} 35 \\
12,9 \%\end{array}$ & $\begin{array}{l}24 \mathrm{~m} 35 \\
10,7 \%\end{array}$ & $\begin{array}{l}0 \\
0 \%\end{array}$ & $\begin{array}{l}2 \mathrm{~h} 03 \mathrm{~m} 55 \\
17,2 \%\end{array}$ & $\begin{array}{l}21 \mathrm{~m} 55 \\
12,71 \%\end{array}$ & $\begin{array}{l}1 \mathrm{~h} 06 \mathrm{~m} 40 \\
21,26 \%\end{array}$ & $\begin{array}{l}49 \mathrm{~m} 40 \\
13.27 \%\end{array}$ & $\begin{array}{l}27 \mathrm{~m} 30 \\
21,4 \%\end{array}$ & $9 / 10$ \\
\hline
\end{tabular}

\section{Annexe 2}

Tableau synthèse des résultats (temps d'enseignement des savoirs mobilisés pendant la lecture analytique)

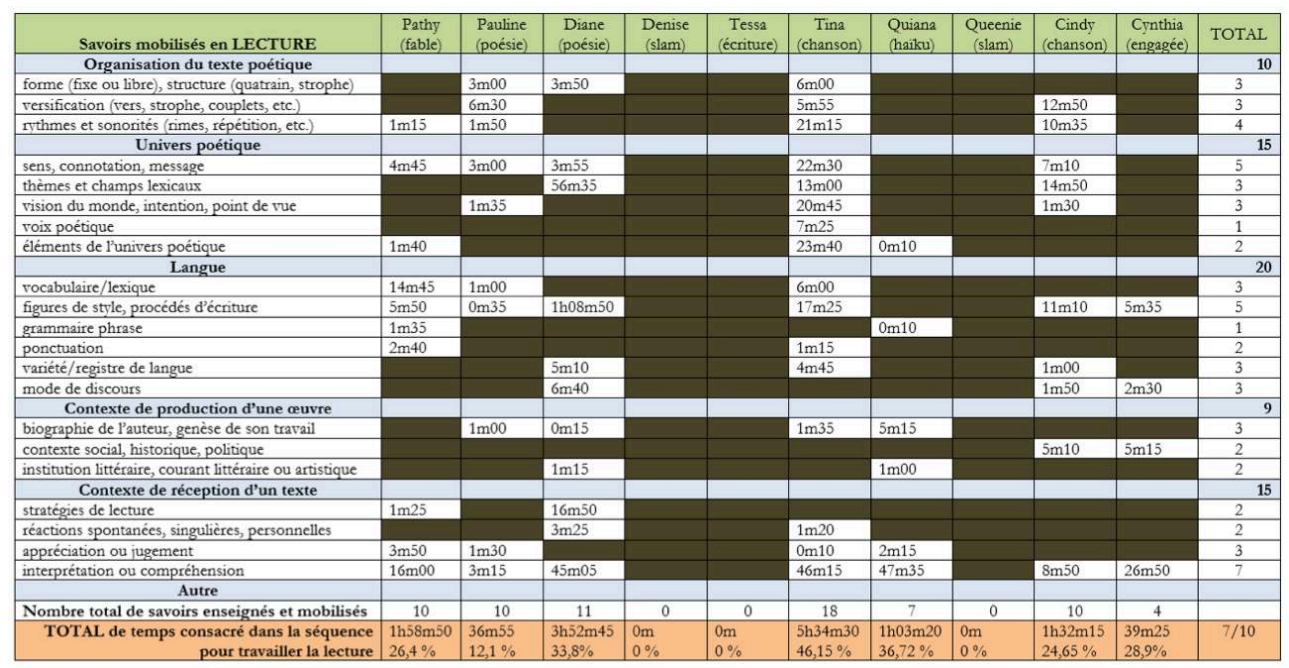

\section{NOTES}

1. Nelsen (1960, p. 16). 
2. Cette recherche a pu être réalisée grâce au financement du Conseil de recherche en sciences humaines (CRSH, 2016-2019). Je tiens en outre à remercier mes étudiantes Amélie Lafrance et Camille $\mathrm{S}$. Leclerc qui ont collaboré à la collecte et à l'analyse des données.

3. Les résultats issus de ces entretiens ont déjà été diffusés : Émery-Bruneau (2018a ; 2018b).

4. Rappelons que les premières Rencontres des chercheurs en didactique de la littérature se sont tenues en 2000 à Rennes.

5. Colloque tenu à Rennes en janvier 2004.

6. Il s'agit d'un échantillon de convenance. Après avoir été sollicités par un courriel envoyé à tous les enseignants de français de huit différentes commissions scolaires, les enseignants qui ont accepté de participer à notre recherche l'ont fait sur une base volontaire.

7. Nous nous inscrivons ainsi dans une approche méthodologique similaire à celle de B. Schneuwly et J.Dolz (2009) qui ont cerné les noyaux durs de séquences d'enseignement de la relative (13 séquences observées) et de textes argumentatifs (17 séquences observées), ainsi que les travaux sur l'analyse des pratiques de formation en écriture, où J. Dolz et R. Gagnon (2018) ont analysé 10 séquences de formation pour l'enseignement au secondaire et 18 pour le primaire.

8. Au Québec, la durée des cours au secondaire est régie par la direction de l'établissement ou la commission scolaire. Pour les classes ordinaires, la durée d'une séance est de 75 minutes en général, ou parfois de 60 minutes. Il en est de même des concentrations enrichies, par exemple le programme de sport-études, qui réduisent la durée des séances (par exemple 50 minutes) afin d'aménager les horaires des élèves pour qu'ils puissent s'entrainer. C'est ce qui explique cette variation dans la durée des séances observées.

9. Parfois la séance commençait quelques minutes après la cloche, ou se terminait plus tôt (pour consacrer le reste de la séance à un tout autre objet), ce qui explique que les totaux des heures/minutes varient des totaux institutionnels.

10. Dans le tableau 2, les durées sont traduites en pourcentage, de façon à mieux saisir les proportions de temps d'enseignement au sein de chaque séquence. Il ne s'agit pas de calculs statistiques, mais plutôt d'un outil pour orienter l'analyse et faciliter les comparaisons au sein d'une même séquence et entre les séquences.

11. Dans toutes les figures présentées ici, nous avons tenté de mettre approximativement à l'échelle les cases de façon à représenter les proportions qu'occupe chaque phase de la séquence. Par exemple, dans la figure 1, le temps de transmission de savoirs est moindre que celui consacré à l'analyse de textes.

12. Ces évènements sont souvent gratuits et ils existent dans la plupart des régions du Québec. En outre, depuis 2019, dans la foulée de la politique culturelle du Québec Partout, la culture et du Plan d'action gouvernemental en culture, un soutien financier est accordé dans le but de permettre à tous les élèves, du préscolaire à la fin du secondaire, de participer à deux sorties culturelles gratuites chaque année, comme aller au théâtre, au musée, au salon du livre, etc. Rappelons par ailleurs que parmi les pratiques culturelles des futurs enseignants, comme assister à un spectacle de théatre ou de musique, l'art oratoire (conte, légende, slam, soirée de poésie...) s'inscrit au dernier rang (Lépine \& Bélanger, 2021, Sous presse), alors raison de plus de provoquer 
ces rencontres pour ouvrir élèves, futurs enseignants et enseignants à des pratiques culturelles variées, dont des pratiques poétiques contemporaines.

13. Université du Québec à Trois-Rivières (LNG1070 : Le langage poétique) ; Université du Québec à Rimouski (MET10110: Approches de la poésie); Université du Québec à Montréal (LIT6235 : Histoire et esthétique de la poésie).

14. Université du Québec en Abitibi-Témiscamingue (LIT1226: Contes, poésie et chanson) ; Université Laval (LIT1908 : Lire la poésie et le théâtre).

\section{RÉSUMÉS}

Nous avons observé des pratiques ordinaires d'enseignement de la poésie dans des classes du secondaire québécois (élèves de 12 à 17 ans) afin d'analyser les savoirs et les pratiques langagières qui constituent le noyau central de cet objet. Trois questions ont guidé nos analyses après avoir produit les synopsis de 10 séquences d'enseignement mises en place par les enseignants (composées de 3 à 13 séances, selon les cas). Quelles sont les trames prototypiques dans les pratiques d'enseignement de la poésie au secondaire québécois ? De quoi est constitué le noyau dur de cet enseignement? Quels lecteurs de poésie forme-t-on? Les résultats montrent que les pratiques d'enseignement actuelles de la poésie visent peu à faire vivre des expériences poétiques; on cherche plutôt à transmettre et à faire pratiquer des savoirs techniques dans le but de les repérer dans les poèmes ou de les reproduire à l'écrit. Nous concluons par une invitation à penser à une didactique qui ne serait pas réduite qu'à la production de savoirs, mais qui serait aussi créatrice d'expériences.

We observed poetry teaching practices in Quebec highschool classes (students ages 12 to 17) to analyze the knowledge and language practices constituting the core of this object. Three questions guided our analysis of the synopsis of 10 teaching sequences developed by the teachers (composed of 3 to 13 sessions, depending on the case): What are the prototypical frames in the poetry teaching practices in Quebec highschools? What is the core of the teaching? What types of poetry readers are we developing? The results show that the poetry teaching aims less at the poetic experiences of students and more at transmitting and practicing technical knowledge that students can identify in poems or reproduce in writing. We conclude with an invitation to think about a didactic that produce knowledge as well as creative experiences.

\section{INDEX}

Mots-clés : didactique, littérature, poésie, lecture littéraire, pratique d'enseignement, progression, expérience poétique

Keywords : didactic, literature, poetry, literary reading, teaching practices, progression, poetic experiences 
AUTEUR

JUDITH ÉMERY-BRUNEAU

Université du Québec en Outaouais, QC J8X 3X7, Gatineau (QC), Canada 